\title{
Reflets
}

Revue d'intervention sociale et communautaire

\section{Les mémoires de maîtrise en service social à l'Université d'Ottawa et à l'Université Laurentienne}

\author{
Ariana M. Anam, Catherine-Anne Bissinger, Brigitte Cadieux, Josette Cattan, \\ Melanie Ciccone, Aminata Diallo, Julie Guérin, Nency Héroux, Farah-Anne \\ Jean-Pierre, Mélanie Laberge, Isabelle Lebeau, Josette Leblanc, Lisa-Marie \\ Loeffen, Mathieu Lyons, Caroline Mainville, Maxine Rodier, Ernest Romain, \\ Isabelle Sabourin, Claire Sales, Melissa Saumur, Mylène Sirois, Immaculée \\ Sugira, Chantal Théberge, Caroline Veilleux et Stefanie Wirz
}

Volume 16, numéro 1, printemps 2010

Paternités méconnues (Deuxième partie)

URI : https://id.erudit.org/iderudit/044448ar

DOI : https://doi.org/10.7202/044448ar

Aller au sommaire du numéro

Éditeur(s)

Reflets : Revue d'intervention sociale et communautaire

ISSN

1203-4576 (imprimé)

1712-8498 (numérique)

Découvrir la revue

Citer ce document

Anam, A. M., Bissinger, C.-A., Cadieux, B., Cattan, J., Ciccone, M., Diallo, A., Guérin, J., Héroux, N., Jean-Pierre, F.-A., Laberge, M., Lebeau, I., Leblanc, J., Loeffen, L.-M., Lyons, M., Mainville, C., Rodier, M., Romain, E., Sabourin, I., Sales, C., Saumur, M., Sirois, M., Sugira, I., Théberge, C., Veilleux, C. \& Wirz, S. (2010). Les mémoires de maîtrise en service social à l'Université d'Ottawa et à l’Université Laurentienne. Reflets, 16(1), 214-240.

https://doi.org/10.7202/044448ar 


\title{
La construction de l'identité sexuée chez les jeunes issus de l'immigration : comparaisons exploratoires entre la France et le Canada
}

\author{
Ariana M.Anam \\ École de service social, Université d'Ottawa
}

Le présent mémoire vise à expliquer et à illustrer, de manière exploratoire, les ressemblances et les divergences de deux sociétés (la France et le Canada) dans le traitement que font les chercheurs de la question croisée du genre et de l'ethnicité chez les jeunes d'origine immigrée. Ce portrait sera établi en puisant dans l'histoire de l'immigration en France et au Canada ainsi que dans leurs modèles respectifs d'intégration des immigrants.

De plus, l'aspect comparatif de cette étude exploratoire sur le sujet de la construction de l'identité de genre portera sur les croisements conceptuels et les rapports entre genre, ethnicité et seconde génération. De fait, les références multiples et mondes sociaux auxquels sont soumis les adolescents issus de l'immigration en France et au Canada (univers familial, institutions éducatives, groupe de pairs, etc.) peuvent être contradictoires et/ou complémentaires.

Toutefois, ces valeurs incitent forcément ces jeunes à jongler de façon à ce qu'ils construisent leur identité de genre de manière active avec ce qu'ils jugent cohérent et significatif. Enfin, cette construction liée à celle du genre prend en compte des différences entre les sexes et les inégalités sociales qui en découlent. 


\title{
Analyse critique de la réforme des services de santé mentale en Ontario (revue de littérature)
}

\author{
Catherine-Anne Bissinger \\ École de service social, Université d'Ottawa
}

Trente ans après la déclaration d'Alma-Ata (1978), la santé mentale en Ontario, comme ailleurs, continue à être négligée, suscitant ainsi des répercussions pour les individus, les familles, les communautés et pour les systèmes de santé. D'où l'importance de prendre un recul sur les projets de réforme des services en santé mentale dans cette province canadienne. Ce mémoire présente une revue des écrits sur la réforme en santé mentale en Ontario publiés après 2000. Les études consultées discutent les stratégies de réforme et de l'orientation des changements du domaine de la santé mentale. Les thèmes qui reviennent le plus souvent dans notre corpus des écrits sont les suivants : l'importance d'augmenter le rôle du client, l'importance d'augmenter la collaboration entre les professionnels, la gouvernance locale des services de santé mentale et le passage aux services de santé mentale communautaires, les obstacles à l'utilisation des services de santé mentale puis le recours aux mécanismes de marché dans la réforme en santé mentale. D'ailleurs, le rapport gouvernemental Franchir les étapes du ministère de la Santé et des Soins de longue durée a été analysé sous l'angle de ces thèmes. 


\title{
Arts martiaux, valeurs et intervention sociale innovatrice : un ménage à trois gagnants pour contrer la violence
}

\author{
Brigitte Cadieux \\ École de service social, Université d'Ottawa
}

Le travail social pensé autrement, la passion pour les arts martiaux, la notion de valeurs comme fil conducteur, les jeunes comme clientèle cible, voilà un mélange d'intérêt et de passion qui a guidé notre sujet de mémoire, dont le propos s'étale sur quatre chapitres. Le premier chapitre sert d'introduction tandis que le deuxième traite de la problématique de la violence chez les jeunes d'âge scolaire. La problématique y est expliquée sous un angle sociologique tout en jetant un regard sur ses formes et ses conséquences. Dans le troisième chapitre, nous développons notre cadre d'analyse qui a été orienté par la notion de valeurs. La question de fond se formule ainsi : "Comment les arts martiaux peuvent-ils outiller les jeunes dans l'acquisition de valeurs saines? " Enfin, un quatrième chapitre dresse un bref historique des arts martiaux traditionnels et de leur apport en intervention sociale. Y figure un tableau sur les résultats de recherche, lequel résume les points saillants de certains d'entre eux. Nous concluons notre étude par l'affirmation qu'arts martiaux, valeurs et intervention sociale innovatrice constituent un bon ménage à trois pour contrer la violence chez les jeunes. 


\title{
La résilience chez les Autochtones. Culture, identité et guérison : le chemin vers un meilleur futur
}

\author{
Josette Cattan \\ École de service social, Université d'Ottawa
}

Longtemps décrite comme une qualité individuelle, la résilience est vue dans le cadre de ce mémoire comme un processus continu et changeant. Ainsi, l'aspect relationnel du processus de résilience sert à expliquer la persistance des Autochtones à continuer d'exister malgré la présence de plusieurs séquelles de la colonisation. Cette persistance peut s'expliquer comme une expression de culture et de cheminement positif visant un avenir meilleur pour les communautés.

L'objet de ce mémoire est de présenter les éléments de résilience qui permettent aux Autochtones de continuer à persévérer malgré l'adversité. Nous démontrerons que la culture est un élément de protection dans le processus de résilience qui promeut le développement de l'identité vers une meilleure conception de soi et du monde. Cela entraine une continuité de soi et des communautés, créant ainsi une vision commune pour un changement social.

Afin de promouvoir ce processus de résilience, il est important de mettre l'accent sur ce qui se révèle comme positif dans les communautés autochtones. C'est pourquoi il est nécessaire de faire ressortir les forces et les ressources des individus durant les interventions ou les cérémonies traditionnelles. Les recherches doivent, elles aussi, porter sur ce principe afin de se concentrer sur l'identification des éléments qui peuvent renforcer et améliorer les liens entre les membres et les communautés autochtones et ainsi persévérer leur culture dans leur processus de résilience. Nous pensons que c'est le concept du «capital social » qu'il serait pertinent d'adopter pour suivre une telle piste dans les recherches.

Enfin, le concept de la résilience repose sur la reconnaissance des diversités culturelles afin de mieux comprendre les éléments 
de certains groupes ou individus qui leur permettent de continuer d'exister malgré les changements rapides dans notre société. Ainsi, en identifiant les composantes culturelles des différents groupes, nous pouvons mieux cerner les éléments qui doivent êtres encouragés dans le processus de résilience.

\section{Remettre en question des pratiques professionnelles : analyse d'approches utilisées lors $d^{\prime}$ 'interventions auprès des personnes atteintes $d^{\prime}$ une maladie chronique}

\section{Melanie Ciccone \\ École de service social, Université d'Ottawa}

Le but de notre recherche est de démontrer qu'il existe diverses façons d'intervenir auprès de gens atteints de maladies chroniques, mais que seulement certaines formes d'intervention, telles que l'approche structurelle et l'intervention de groupe, peuvent promouvoir l'action sociale.

Dans le cadre de ce mémoire, nous privilégions une analyse documentaire. Ce type d'analyse nous permet d'explorer le sujet des maladies chroniques et d'identifier les différents enjeux qui entourent ce phénomène pour ensuite faire l'analyse de certaines approches utilisées par des travailleuses sociales et des travailleurs sociaux qui œuvrent auprès des gens atteints de maladies chroniques. Les trois approches étudiées sont l'approche cognitivo comportementale, le service social des groupes et l'approche structurelle.

Les résultats de notre analyse nous permettent de cibler les différences entre les objectifs d'intervention des approches choisies, le rôle entrepris par les travailleuses sociales et les travailleurs sociaux, ainsi que les limites et les forces des approches. Nous avons ainsi déterminé la façon par laquelle les facteurs comportementaux 
et les facteurs structurels ont des conséquences sur la santé des individus. Bref, cette analyse nous a permis de constater que les personnes qui sont plus à risque d'une santé appauvrie sont affectées par divers facteurs structurels. Afin de remettre en question les facteurs structurels et de corriger les limites de l'approche cognitivo comportementale et du service social des groupes, nous présentons en guise de solution, une approche structurelle implantée à l'intérieur d'un groupe.

\title{
Femmes enceintes, mères et attachement maternel en contexte de toxicomanie : une parentalité escamotée
}

\author{
Aminata Diallo \\ École de service social, Université d'Ottawa
}

La société occidentale nourrit à l'égard des femmes un certain nombre d'attentes, d'attitudes, de comportements et de rôles. Certaines attentes sont de nature biologique comme la procréation et l'allaitement. Par ces comportements, nous attendons que les femmes prennent soin de leurs enfants, qu'elles les protègent des malheurs possibles et qu'elles leur assurent un développement physique et psychique approprié. Nous parlons donc ici de leur parentalité. L'objectif de ce mémoire est d'explorer les capacités parentales des mères lorsqu'elles ont une dépendance aux substances illicites dures. Nous nous centrons sur la capacité de ces mères d'offrir des soins physiques et affectifs au travers d'une analyse des différents axes de la parentalité et des facteurs qui favorisent un meilleur attachement. Cette analyse se fait parallèlement à la situation des femmes non consommatrices de substances illicites afin de comprendre l'ampleur exacte de cette problématique. 


\title{
Analyse documentaire des ouvrages de Jean-Claude Kaufmann (1983-2009) et de sa position face au couple contemporain
}

\author{
Julie Guérin \\ École de service social, Université d'Ottawa
}

Le présent mémoire consiste en une analyse documentaire qui porte sur les théories élaborées par Jean-Claude Kaufmann, sociologue français. Il se distingue par son approche originale d'enquête de terrain qui explore la signification des microgestes du quotidien. Ce mémoire est structuré en deux parties. La première partie présente, en ordre chronologique, un résumé de dix-sept ouvrages de l'auteur publiés entre 1983 et 2009. Cette approche permet de repérer les grandes articulations de sa pensée et de saisir comment, au fil des enquêtes, il est arrivé à mettre au point un cadre théorique sociophilosophique. Cette présentation permet également de comprendre pourquoi et à quel moment il s'est intéressé au couple contemporain, dont il est devenu un spécialiste reconnu. La deuxième partie consiste en une analyse croisée de ses trois ouvrages clés sur le couple, lesquels examinent la trajectoire particulièrement précaire et improvisée des individus, de la rencontre initiale à l'installation conjugale. Dans La trame conjugale, Kaufmann analyse comment le partage des tâches est particulièrement problématique et conduit au maintien des inégalités entre les sexes. Dans Premier matin, les automatismes culturels des deux partenaires qui se confrontent lors de leur première rencontre sont expliqués comme étant des obstacles à la découverte intime de l'autre. Des stratégies individuelles se développent en fonction des craintes et des attentes de chacun. Le défi principal que rencontrent les individus est la peur de l'abandon de soi pour aller vers l'autre. Agacements analyse les différences individuelles qui sont responsables de nombreuses dissonances entre les partenaires. Il analyse également les mécanismes de régularisation du couple et l'apport de chacun 
des individus à l'intérieur de la sphère conjugale. Le couple est certes devenu plus difficile à construire, mais les individus sont plus aptes à vaincre les difficultés en portant une plus grande attention aux contradictions qui surgissent inévitablement lorsque deux sensibilités s'affrontent.

\title{
Le travail social dans le réseau de la santé et des services sociaux et les effets de la nouvelle gestion publique : implications cliniques et choix organisationnels
}

\author{
Nency Héroux \\ École de service social, Université d'Ottawa
}

La proposition initiale de cette recherche porte sur la Nouvelle Gestion publique (NGP) et ses effets sur la pratique des travailleurs sociaux. Les résultats exposent l'aspect préoccupant d'une organisation de services fondée d'abord et avant tout sur des cibles de gestion. L'objectif de cette recherche est de comprendre les enjeux et les impacts de la NGP pour la pratique du travail social, en particulier dans les services de soutien à domicile. Il s'agit d'une recherche qualitative utilisant l'approche narrative par le biais d'un récit de pratique. Le principal moyen utilisé pour la collecte des données a été le journal de bord. Les thèmes ayant fait l'objet d'observations et de réflexions systématiques sont la pratique du travail social et l'organisation du travail dans un service de soutien à domicile d'un CSSS de grande taille. L'allocation des ressources financières dans les établissements publics au Québec est maintenant fonction de l'atteinte de cibles de gestion (MSSS, 2005). Cette reddition de compte s'inscrit clairement dans les principes de la NGP : l'efficience et la performance en sont le leitmotiv. Bien qu'ils soient responsables de s'assurer que les deniers publics soient utilisés à bon escient, les établissements 
publics transforment rapidement cette vigilance en élément de dysfonction lorsque ce sont les cibles de gestion qui gouvernent et orientent les décisions sur l'organisation des services à offrir à la population. Merrien (1999) l'exprime ainsi : «L'erreur fondamentale de la Nouvelle Gestion publique est de porter les responsables à s'intéresser prioritairement à l'efficience de leur organisation en négligeant la réponse aux besoins de la société.» (Merrien, 1999, p.101). La démarche d'analyse confirme non seulement que l'exercice du travail social est heurté dans son essence première, la relation d'aide, à la suite de l'introduction du diktat de la rentabilisation des interventions, mais témoigne également de l'incidence réelle de la NGP sur l'organisation des services à la population. Alors qu'il est entendu que les services de santé et les services sociaux publics doivent correspondre aux besoins de la population, les résultats de cette recherche amènent à douter de cette allégation.

\title{
Le mariage forcé : état de la situation
}

\author{
Farah-Anne Jean-Pierre \\ École de service social, Université d'Ottawa
}

Ce mémoire porte sur le mariage forcé et son objectif principal a été de proposer des mesures qui pourraient être prises afin de contrer ce phénomène et ainsi venir en aide aux personnes menacées ou victimes de mariage forcé au Canada, où le phénomène est peu documenté et discuté. Nous avons choisi d'entreprendre une recherche documentaire à partir de plusieurs bases de données électroniques disponibles à l'Université d'Ottawa et à partir de moteurs de recherche de l'Internet. Les sites gouvernementaux de différents pays, en particulier européens, nous ont aussi permis de documenter les lois et les mesures que ces pays ont prises en matière de mariage forcé.

Dans un premier temps, le mémoire définit ce qu'est le mariage forcé en le distinguant du mariage arrangé. Il analyse 
ensuite les principales causes du mariage forcé et ses conséquences sur les victimes et leur entourage, dans les pays occidentaux et dans les pays non occidentaux. Nous constatons alors que les causes du mariage forcé dans les pays occidentaux et dans les pays non occidentaux divergent beaucoup et que la notion de consentement libre et éclairé est à la base des dispositifs pris par les États pour combattre le phénomène. Dans un troisième chapitre, sont identifiés les dispositifs juridiques, les types d'intervention et de prévention dont certains pays se sont dotés pour contrer le problème. En conclusion, le mémoire propose quelques recommandations concernant le mariage forcé au Canada, sujet qui est très peu abordé sur le plan des politiques autant que sur celui de l'intervention. Le mémoire met cependant en garde les décideurs et les intervenants contre des mesures qui pourraient se révéler stigmatisantes envers certaines communautés ethniques originaires des pays du Sud et recommande de travailler avec les communautés les plus concernées afin de trouver des moyens permettant de contrer le mariage forcé.

\section{S'occuper d'un enfant atteint du cancer. Quels effets sur les pères et les mères?}

\section{Mélanie Laberge}

École de service social, Université d'Ottawa

Le cancer touche malheureusement plusieurs enfants et leur famille. Les pères et les mères de ces enfants doivent inévitablement en subir les effets, mais de façons différentes. Cela n'a rien d'étonnant, puisque l'histoire des rapports entre les hommes et les femmes dans notre société est traversée par de grandes inégalités entre les sexes.

Ces inégalités font en sorte que la mère est généralement celle qui s'occupe des enfants et des personnes malades. Les mères seraient donc plus affectées par les effets du cancer d'un enfant et 
subiraient plus d'effets négatifs que les pères. Ce mémoire tente de confirmer cette hypothèse en s'intéressant à la socialisation, aux rôles de genre traditionnels, à la division sexuelle du travail, aux structures sociales, ainsi que sur le plan individuel, aux réactions des mères et des pères face à la situation, aux effets associés à leurs rôles, à leur situation financière, ainsi qu'à leurs stratégies d'adaptation.

Ce mémoire aborde ce questionnement en utilisant une méthode d'analyse de contenu de trente-huit articles scientifiques, de trois livres publiés dans des presses universitaires et d'une évaluation d'un programme de répit à domicile.

L'ensemble de l'analyse montre, entre autres, qu'il est primordial d'adopter des politiques gouvernementales qui tiennent compte de ces différences, notamment en regard de la structure du marché du travail, et d'adapter les interventions afin de mieux aider les familles et diminuer les effets négatifs des inégalités sociales de sexe qui apparaissent au moment de s'occuper de l'enfant malade. Finalement, ce mémoire souligne l'importance de changer certains fondements de la société qui créent des inégalités sociales et, par le fait même, font en sorte que les pères et les mères sont affectés différemment par le besoin de s'occuper d'un enfant malade. En étant conscient de ces différences et de leurs causes, il est à tout le moins possible d'alléger une situation pénible pour les parents et leurs enfants. 


\section{La prostitution à travers une analyse de biographies et de récits de jeunes prostituées}

\section{Isabelle Lebeau}

École de service social, Université d'Ottawa

La prostitution est une problématique que l'on retrouve partout dans le monde et qui touche toutes les couches de la société : les hommes, les femmes, les enfants, surtout dans les pays pauvres, mais également dans les pays industrialisés. Plusieurs éléments ajoutent à la problématique de la prostitution : la pornographie incluant l'accessibilité de l'offre et de la demande sur Internet, les petites annonces, les services de massage et d'escortes, et bien d'autres.

Cette analyse de contenu a pour objectif de mieux faire connaître, à l'aide d'une analyse d'autobiographies, la réalité vécue par un nombre important de jeunes filles. La sélection des biographies s'est limitée à celles disponibles au Québec, traduites ou rédigées en français, racontant une histoire de prostitution chez des jeunes filles de moins de vingt ans. Huit monographies et neuf histoires de vie tirées d'un recueil de témoignages d'exprostituées ont servi à l'analyse.

Les résultats de la cueillette de données et de l'analyse relèvent les facteurs de risque et les facteurs de sortie les plus souvent identifiés dans chacun des récits. Parmi les facteurs de risque les plus souvent évoqués, notons : la toxicomanie, les agressions sexuelles, la violence tant physique que psychologique, et la pauvreté qui, malgré qu'elle soit un facteur omniprésent dans la grande majorité des récits, ne contribue pas à elle seule à amener les jeunes vers la prostitution. Toutefois, ces facteurs regroupés peuvent engendrer chez une jeune personne fragilisée une vulnérabilité accrue. Quant aux facteurs de sortie, les plus souvent cités sont la maladie physique, la grossesse, la dépression et l'aide de la famille. 


\title{
Les conjointes de militaires : des héroïnes de l'ombre
}

\author{
Josette Leblanc \\ École de service social, Université d'Ottawa
}

Cette recherche présente les résultats d'une analyse documentaire qui porte sur la réalité des conjointes de militaires et sur les stratégies d'ajustement (coping) qu'elles utilisent pour pallier les nombreux défis imposés par le mode de vie militaire. À partir d'une recherche bibliographique et de réflexions provenant d'observations informelles, nous avons procédé à un examen préliminaire des documents recensés afin d'identifier les plus pertinents à notre recherche. Pour faciliter l'analyse, nous avons procédé à un classement des documents d'abord selon le thème (vécu ou stratégie d'ajustement) et ensuite selon la source, le type et le contexte. Selon les résultats de notre analyse, la situation des conjointes de militaires a fait l'objet de peu d'études canadiennes, mais le milieu universitaire commence à s'y intéresser davantage. De plus, certaines caractéristiques du mode de vie militaire contribuent à l'isolement et à une vulnérabilité économique et sociale de ces femmes : les fréquents déménagements, la culture militaire, les barrières linguistiques, l'imprévisibilité, la notion d'être prêt au combat (combat ready), etc. La culture militaire est fortement imprégnée par le côté hiérarchique et patriarcal de l'armée, ce qui influence et détermine le rôle et la place des conjointes au sein de l'organisation militaire. Ces femmes vivent une réalité qui exige d'importants sacrifices sur le plan personnel et professionnel, et doivent mettre en pratique des stratégies d'ajustement (coping) pour pouvoir survivre dans ce type d'environnement. Les documents révèlent une réalité empreinte de défis à relever et de problématiques particulières à résoudre. L'intervenant qui travaille auprès de cette clientèle doit être à l'affût de ce qui se passe dans la communauté militaire et ajuster ses interventions en conséquence. 


\section{Programme d'intégration pour jeunes immigrantes et immigrants de $\mathbf{1 2}$ à $\mathbf{1 6}$ ans au sein des écoles francophones d'Ottawa}

\section{Lisa-Marie Loeffen}

École de service social, Université Laurentienne

Dans le cadre de ce mémoire, nous tentons de comprendre le phénomène de l'intégration à une nouvelle culture des adolescentes immigrantes et des adolescents immigrants d'expression française, âgés de douze à seize ans, de la ville d'Ottawa. Tout particulièrement, l'objectif du mémoire est de développer un programme qui pourrait faciliter l'intégration scolaire et sociale des jeunes immigrantes et immigrants âgés de douze à seize ans en milieu scolaire francophone dans la ville d'Ottawa. Les données recueillies par l'entremise de cette recherche permettent une meilleure compréhension des défis que vivent les adolescentes et adolescents qui tentent de s'intégrer à un nouveau milieu. Ces données offrent une source d'informations pour les pourvoyeurs de services sociaux, les conseils scolaires et les responsables d'élaboration de politiques en milieux social et scolaire. Le programme développé par l'entremise de ce mémoire fournit l'information nécessaire pour que les conseils scolaires et les organismes de services sociaux communautaires puissent développer en partenariat ce programme et le mettre en ouvre. 


\title{
Le rôle paternel : l'influence de l'homophobie sur la paternité
}

\author{
Mathieu Lyons \\ École de service social, Université d'Ottawa
}

Ce mémoire a pour objectif d'étudier l'influence de l'homophobie sur l'engagement paternel de pères en situation de vulnérabilité. Il se base principalement sur des entrevues réalisées auprès de jeunes pères. Il est constitué de six sections. L'hétérosexisme et les types d'homophobie sont définis dans la première. Dans la section suivante, l'hétérosexisme et l'homophobie sont étudiés sous une dimension historique en situant le positionnement actuel de l'Église, celui des grandes institutions de la santé et celui des institutions législatives. Suivra une réflexion sur la définition de la paternité qui dressera un bref rappel historique de l'évolution du rôle de père en plus de présenter les définitions contemporaines de l'engagement paternel. L'influence de la vulnérabilité socioéconomique sur l'exercice de la paternité boucle cette partie.

Par la suite, nous esquisserons le processus de socialisation des hommes au sein de la société nord-américaine et son influence sur la façon d'être père. Puis, après avoir expliqué la méthodologie utilisée dans le cadre de notre recherche, nous exposerons les résultats des entrevues, afin de répondre à notre question portant sur la définition du rôle paternel des répondants en lien avec l'influence de l'homophobie. La dernière section de ce mémoire, la discussion, aura comme objectif de vérifier si l'homophobie générale influence la construction du rôle paternel des jeunes pères et, le cas échéant, de quelles façons. Les trois thèmes traités dans le cadre de la présentation des données y sont analysés : les perceptions des participants face au rôle paternel, leurs perceptions face aux rôles parentaux ainsi que la paternité en lien avec l'homophobie. Nous terminerons avec la présentation de la contribution que cette étude pourrait apporter aux connaissances en travail social. 


\title{
La stratégie de réduction de la pauvreté ontarienne : du pareil au même ou une voie d'avenir?
}

\author{
Caroline Mainville \\ École de service social, Université d'Ottawa
}

Une nouvelle approche aux politiques sociales a été élaborée à la fin du $\mathrm{XX}^{\mathrm{e}}$ siècle et est devenue au début du $\mathrm{XXI}^{\mathrm{e}}$ siècle l'approche privilégiée par plusieurs gouvernements. L'approche se veut au-delà du débat entre la droite et la gauche, se veut différente de ce que l'histoire a déjà connu et se veut alors, un stimulant changement. L'évolution des politiques sociales au Canada et en Ontario a démontré que le débat entre les deux approches aux politiques sociales, le néolibéralisme et la social-démocratie, ne menait plus à de grands progrès à l'égard de la pauvreté. En effet, lors de leur apogée respectif dans l'histoire, la pauvreté existait toujours, et ce, de façon constante. Or, une nouvelle approche était essentielle afin d'avoir l'espoir d'un réel impact sur la pauvreté au Canada.

L'évolution des politiques sociales au Canada et en Ontario a aussi démontré que la pauvreté n'a jamais réellement fait partie du discours politique dans sa globalité. Par contre, en 2008, le gouvernement ontarien de McGuinty a présenté une Stratégie globale de réduction de la pauvreté pour sa province. Cette nouvelle attention à l'égard de la pauvreté reflète l'approche de l'investissement social, car, tout comme la social-démocratie, la protection des plus faibles est parmi les obligations de l'État; mais, à l'opposé de celle-ci, c'est en termes d'exclusion et d'intégration qu'on aborde ces inégalités. Ces termes se trouvaient dans le discours du gouvernement McGuinty avant l'adoption de la Stratégie antipauvreté. Par ce fait, on espérait que la Stratégie qui suivrait les aurait aussi, en plus des autres principes de base de l'approche de l'investissement social. Dans ce mémoire, nous avons analysé la nouvelle Stratégie de réduction de la pauvreté ontarienne. De plus, nous avons approfondi l'analyse en étudiant 
l'histoire des politiques sociales canadienne et ontarienne afin de pouvoir identifier la présence d'autres approches dans le discours et les actions prévues de la Stratégie ontarienne. Une deuxième perspective à la Stratégie provinciale a aussi pu être réalisée en recherchant dans les publications d'un réseau d'organismes à but non lucratif, 25 in 5 . En somme, nous avons montré qu'une nouvelle approche se retrouve dans la Stratégie et dans les actions portées par le gouvernement depuis son adoption, et ce, malgré la crise économique qui a frappé le monde entier. Il est trop tôt pour savoir si cette Stratégie aura une incidence sur les taux de pauvreté en Ontario. Mais puisque l'approche est différente de ce qui a déjà été fait et qu'elle est plus holistique et réfléchie, les futurs résultats seront encourageants, surtout en raison de l'empreinte du réseau 25 in 5 qu'elle comporte.

\title{
Le genre comme déterminant de la santé : une question d'inégalité sociale
}

\author{
Maxine Rodier \\ École de service social, Université d'Ottawa
}

Les inégalités en santé entre les hommes et les femmes sont toujours présentes dans notre société. Les femmes vivent plus longtemps que les hommes, mais elles sont en moins bonne santé. Le genre est un facteur important dans la construction de ces inégalités en santé entre les hommes et les femmes. D'après notre recherche bibliographique des études faites au Canada, il existe trois types de déterminants de la santé, soit les déterminants structuraux, comportementaux et psychosociaux qui agissent de manière différente chez les hommes et les femmes. D'après les études consultées, la vulnérabilité et l'exposition des hommes et des femmes à ces déterminants different, mais aucune des hypothèses ne permet d'expliquer à elle seule les inégalités sociales en santé. Par contre, les connaissances actuelles sur les inégalités sociales 
en santé selon le genre permettent de mettre de l'avant différents types d'interventions afin de diminuer ces inégalités. Ainsi, le travail social a sa place dans la lutte contre les inégalités sociales en santé selon le genre en contribuant à renforcer les individus, à renforcer les communautés, à améliorer les conditions de vie et de travail et en faisant la promotion de pratiques saines.

\section{Vieillissement, religion et spiritualité : défi pour le service social}

\section{Ernest Romain}

École de service social, Université d'Ottawa

Ce mémoire portant sur le vieillissement, la religion et la spiritualité est de type théorique. Ce travail de recherche a pour but de réfléchir sur la place qu'occupent la religion et la spiritualité dans la vie des personnes ainées. Par la suite, il s'attarde à conscientiser les professionnels des services sociaux et de la santé à connaître et à prendre en charge les dimensions religieuses et spirituelles dans leurs interventions auprès des ainés.

Pour ce faire, nous avons utilisé une méthodologie de recherche basée sur l'analyse de contenu documentaire, renforcée par celle de l'observation directe. Cette stratégie a permis de collecter des données sur le vieillissement démographique, les principales religions du monde, les grandes formes de spiritualité et quelques modèles de santé/maladie.

Cette recherche a révélé que le vieillissement entraine partiellement une quête de spiritualité et que, même apte à retarder une série de maladies, la médecine scientifique reste toujours incapable d'aider les ainés en situation de fragilité à donner une signification à leur vie. Cette quête de sens peut être assurée par le biais des pratiques religieuses et spirituelles, ou des deux. Les sciences modernes, surtout la médecine, sont encore lentes à s'ouvrir à la religion et à la spiritualité. Cette étude ouvre donc la 
voie à de nouvelles pistes de recherche et oriente les intervenants sociaux vers une forme d'intervention adaptée à la réalité globale des personnes ainées.

\title{
La fratrie : une expérience essentielle ou en état de disparition? Analyse de la fratrie à travers la littérature des jeunes de 10 à 13 ans
}

\author{
Isabelle Sabourin \\ École de service social, Université d'Ottawa
}

L'objectif de ce mémoire est d'étudier la relation entre frères et sœurs à travers la littérature pour jeunes de 10 à 13 ans. Cette étude est basée sur l'analyse de romans populaires publiés dans les dix dernières années et qui sont comparés à des romans publiés dans les années précédant les années 1950 et qui sont toujours populaires aujourd'hui.

Nous définirons tout d'abord les relations fraternelles. Nous analyserons l'impact de différents écrits historiques sur cette définition et sur les concepts contemporains qui en découlent. Nous explorerons l'impact des relations frères et sœurs sur les différents membres de leur famille à travers l'enfance, l'adolescence, la vie adulte et la vieillesse. Nous survolerons aussi certains concepts contemporains tels que la place dans la fratrie, le mythe de la bonne entente et la mémoire familiale.

Puis, une analyse de contenu de six romans permettra de dégager différents thèmes. Les valeurs relevées autour de la fratrie porteront sur l'importance de donner l'exemple, l'entraide, l'alliance, la solidarité, la rivalité et les conflits, la différenciation, le développement de l'identité et les valeurs de l'amitié.

Parmi les divers éléments de la vie familiale, la fratrie passe souvent inaperçue. Peu de chercheurs réfléchissent au fait que cette relation entre frères et sœurs est généralement la première 
relation sociale sur laquelle se fondent toutes les autres interactions. Avec le nombre croissant de familles à enfant unique, il y a lieu de s'interroger sur ces mêmes interactions. À la suite d'une exploration des études scientifiques sur le sujet, nous analyserons les relations fraternelles à travers le temps dans la littérature destinée aux enfants de 10 à 13 ans. En comparant des romans publiés avant les années 1950 à d'autres écrits durant les dix dernières années, nous tenterons de découvrir les changements survenus dans les familles, et plus spécifiquement dans les fratries.

\section{Le rôle des jardins communautaires dans la sécurité alimentaire chez les Autochtones vivant en milieu urbain}

Claire Sales

École de service social, Université d'Ottawa

Le niveau d'insécurité alimentaire chez les ménages autochtones hors réserve est trois fois plus élevé que celui des ménages non autochtones. Le présent mémoire de recherche examine les raisons de cette statistique étonnante, ainsi que des solutions possibles. Pour ce faire, cette recherche utilise l'approche " analyse de contenu " en examinant les documents écrits sur le sujet et en les présentant à travers certains sous-thèmes. Nous commençons avec le passé en décrivant les valeurs et pratiques traditionnelles des peuples autochtones et les effets de la colonisation sur l'alimentation de ces populations. Ensuite, nous observons l'état de bien-être et de sécurité alimentaire chez les Autochtones vivant en milieu urbain aujourd'hui. Dans cette section, on considère l'importance de la dimension culturelle de la sécurité alimentaire, y compris les aliments et pratiques traditionnels des Autochtones. Avec l'objectif d'explorer les raisons du taux élevé d'insécurité alimentaire chez les Autochtones vivant en milieu urbain, nous étudions l'impact des jardins communautaires urbains 
sur la santé holistique, la sécurité alimentaire et le développement communautaire des communautés autochtones. Cette section se base sur trois études de cas de jardins communautaires autochtones qui existent à Vancouver, Winnipeg et Toronto. Finalement, ce travail présente l'importance de l'implication du service social dans les sujets abordés, comme l'intervention au niveau communautaire, l'intervention auprès des Autochtones, et la sécurité alimentaire. En plus de démontrer le rôle des jardins communautaires dans le bienêtre des communautés autochtones urbaines, le présent mémoire vise à exposer les injustices auxquelles font face les Autochtones depuis des siècles ainsi que l'importance de la compréhension et de la valorisation des cultures autochtones pour les pratiques en service social ainsi que pour la société en général.

\section{Intervention par le jeu}

Melissa Saumur

École de service social, Université Laurentienne

Les enfants et les adolescentes et adolescents pris en charge par la Société de l'aide à l'enfance sont souvent affectés émotivement et psychologiquement par leur vécu de maltraitance, de négligence ou d'abus. Une grande majorité de ces enfants et de ces jeunes peuvent profiter de l'intervention afin d'améliorer leur mieux-être.

Les situations familiales sont souvent très complexes et lorsqu'il y a un parent pour protéger l'enfant ou le jeune, ce dernier peut continuer à vivre avec sa famille d'origine tout en ayant un suivi offert par la Société d'aide à l'enfance. C'est à ces familles que je me suis intéressée dans mon stage spécialisé, pendant lequel, entre autres activités, j'ai fait de l'intervention par le jeu auprès de six enfants et jeunes demeurant avec leur famille.

Durant ce stage, j'ai pu améliorer mes connaissances théoriques sur l'intervention par le jeu et mes habiletés cliniques, et j'ai bénéficié de toute la supervision nécessaire pour mener à bien les interventions avec les enfants et les jeunes. 


\title{
Suivre le sillon de ses parents ou explorer sa voie? Étude exploratoire des parcours d'études de jeunes issus de milieux francophones minoritaires
}

\author{
Mylène Sirois \\ École de service social, Université d'Ottawa
}

Selon diverses enquêtes, les jeunes issus d'un milieu francophone minoritaire dont les parents ont obtenu un diplôme d'études postsecondaires accèdent assez facilement aux études universitaires. C'est dans ce contexte que nous avons choisi d'explorer les différences de parcours d'études entre les étudiants dont les parents n'ont pas fait d'études postsecondaires et ceux dont les parents ont obtenu un diplôme de baccalauréat.

Afin de répondre à ce questionnement, ce mémoire analyse les parcours d'études universitaires de 20 étudiants issus d'un milieu francophone minoritaire : 8 d'entre eux ont des parents qui n'ont aucun diplôme d'études postsecondaires tandis que 12 ont des parents qui ont chacun obtenu un baccalauréat. L'analyse de contenu a permis d'identifier six dimensions ayant une influence particulière sur l'accès aux études postsecondaires.

Les résultats de l'étude montrent qu'il existe à la fois des similarités et des différences entre les deux groupes. Les ressemblances sont généralement liées au contexte d'origine, soit, le milieu francophone minoritaire, alors que les différences portent sur le rôle de la famille dans le parcours scolaire ainsi que sur les dimensions financières des parcours des étudiants. Les jeunes dont les parents n'ont pas fait d'études postsecondaires reçoivent moins d'encouragements de la part de leurs parents et de leur famille que ceux dont les parents ont effectué des études postsecondaires. Ces jeunes reçoivent aussi moins de soutien financier, ce qui peut contribuer à fragiliser leurs parcours d'études et causer des situations d'endettement problématiques. Les résultats permettent de proposer certaines pistes d'intervention au sens large au niveau des communautés francophones et du milieu universitaire ainsi que dans le domaine des politiques sociales. 


\title{
Impacts de la nouvelle organisation du travail sur la vie familiale
}

\author{
Immaculée Sugira \\ École de service social, Université d'Ottawa
}

Le travail est un facteur important dans la vie des individus et de toute société humaine. Il est cependant important de reconnaître que l'individu ne vit ni ne travaille dans " le vide », mais bien dans un contexte sociohistorique, politico-économique et culturel plus large, et que son bien-être physique et psychologique est étroitement lié à une coordination satisfaisante entre son travail et sa vie familiale. Quand on pense au rythme de la société actuelle, à la rapidité avec laquelle la famille évolue, à la nouvelle organisation du travail, on ne peut s'empêcher de poser la question capitale : pourquoi l'équilibre entre travail et famille devient-il un enjeu aussi important dans notre société actuelle?

En empruntant la voie d'une recherche documentaire, ce travail de mémoire se propose d'explorer les différents impacts de la nouvelle organisation du travail sur la conciliation travailfamille des parents de jeunes enfants. 


\title{
Étude du discours de jeunes pères sur leur enfance sous l'angle de la théorie d'attachement
}

\author{
Chantal Théberge \\ École de service social, Université d'Ottawa
}

Ce mémoire a pour but d'étudier le regard que des jeunes pères ont sur le lien qu'ils ont connu avec leurs parents. Pour ce faire, les principaux concepts qui constituent la théorie de l'attachement sont expliqués. Par la suite sont présentés les résultats de cette étude réalisée à partir d'entretiens effectués auprès de 17 jeunes pères sélectionnés parmi 30 participants recrutés dans le cadre d'un projet de recherche précédent. Certains indicateurs de l'Entrevue sur l'attachement à l'âge adulte ont été utilisés pour analyser ces résultats. Les résultats portent spécifiquement sur ceux qui rapportent avoir connu une enfance heureuse ou " correcte ", c'est-à-dire passable. Les premiers ont un état d'esprit actuel sécurisé/autonome et ceux décrivant leur enfance comme étant correcte présentent un état d'esprit actuel détaché. Toutefois, d'autres facteurs vécus par ces jeunes pères durant leur jeunesse, tels que la séparation de leurs parents, ainsi que les conflits familiaux, le style de discipline parentale connu durant leur jeunesse et leurs expériences de vie difficiles à l'adolescence, peuvent avoir influencé leur relation d'attachement avec leurs parents. L'analyse des entrevues permet de constater que les jeunes pères décrivant leur enfance comme étant heureuse semblent avoir vécu moins de facteurs de risque en comparaison avec les jeunes pères décrivant leur enfance comme étant correcte. De plus, il ressort de cette étude que la grande majorité de ces jeunes pères, et ce, dans les deux catégories, ont adopté des comportements marginaux à l'adolescence. 


\section{Que signifie être jeune adulte aujourd'hui? : point de vue de jeunes adultes ayant reçu un diagnostic de maladie mentale}

\section{Caroline Veilleux \\ École de service social, Université d'Ottawa}

Ce mémoire s'inscrit dans une démarche d'exploration de la réalité des jeunes adultes ayant reçu un diagnostic de maladie mentale, et ce, à travers la considération de leur discours. Il vise dans un premier temps à déterminer si ces jeunes adultes se perçoivent comme des adultes et à identifier les critères associés à cette perception d'être adulte. En second lieu, le projet cherche à circonscrire l'impact de la maladie mentale sur la perception générale de soi et à connaitre la représentation et le positionnement de ces jeunes adultes par rapport aux services en santé mentale. Pour atteindre ces objectifs de recherche, une méthodologie de type qualitatif a été appliquée. Des entrevues semi-dirigées ont été réalisées auprès de huit jeunes adultes, tous âgés de 18 à 35 ans, qui ont reçu un diagnostic de maladie mentale; une fois transcrites, ces entrevues ont été soumises à une analyse de contenu.

L'analyse approfondie du discours a permis de relever que la majorité des jeunes adultes interviewés se considèrent en partie comme des adultes. Leur perception d'être adulte repose à la fois sur des caractéristiques individuelles telles que la responsabilisation et l'autonomie et sur des marqueurs de transitions comme la mise en couple et l'entrée dans le marché du travail, ce qui rejoint essentiellement la tendance observée chez les jeunes adultes en général. En comparant la réalité des jeunes adultes sans problème de santé mentale avec celle des jeunes adultes interviewés, il a été possible de constater des distinctions marquées sur le plan du rapport à soi et du rapport à autrui. De façon générale, les huit jeunes adultes se réferent moins aux marqueurs de transition formels pour expliquer leur parcours de vie et s'en remettent davantage à leurs expériences personnelles. 
Ils s'investissent davantage dans des activités occupationnelles et des relations interpersonnelles significatives à leurs yeux et tendent à se détacher des pressions et des autres normes sociales, particulièrement au niveau professionnel. De plus, l'expérience de la maladie mentale entraîne d'importantes remises en question identitaires, frappe directement la qualité des rapports familiaux et redirige la définition prospective de soi. Concernant les services de santé mentale, les jeunes adultes interviewés préconisent des interventions humaines de longue haleine qui tiennent compte de leurs réalités plurielles et parallèlement, des pratiques collectives, préventives et dépourvues de tabou et de stigmate.

Ce bref portrait de la réalité de jeunes adultes ayant reçu un diagnostic de maladie mentale et des enjeux qui les touchent, représente une occasion inestimable de leur donner un espace de parole ouvert et reconnu et de stimuler à leur égard la réflexion critique, tant dans la population que dans le réseau de la santé mentale et dans la communauté scientifique.

\section{Les politiques d'admission de réfugiés au Canada. Regard critique sur un système marqué par l'ambiguïté}

Stefanie Wirz

École de service social, Université d'Ottawa

La politique de protection des réfugiés au Canada est marquée par l'ambiguiité. Un certain nombre d'éléments du système actuel d'admission des réfugiés souffre de contradictions et de conflits de valeurs. Ce phénomène est le résultat de l'influence simultanée de deux groupes d'acteurs principaux sur le système : les acteurs institutionnels et les acteurs non gouvernementaux. Les acteurs institutionnels (les gouvernements fédéral et provinciaux et la Commission de l'immigration et du statut de réfugié [CISR]) défendent un discours de souveraineté, en soulignant les intérêts 
économiques et sécuritaires du Canada; ils revendiquent une politique d'admission restrictive. D'un autre côté, les acteurs non gouvernementaux (les réfugiés et les organisations défendant les droits des réfugiés) s'inscrivent dans le discours de l'humanitaire. Ces acteurs s'opposent à la restriction de l'accès à la protection pour les réfugiés et ils revendiquent une politique plus ouverte.

La présente analyse de la politique canadienne de protection des réfugiés récapitule l'historique des politiques des réfugiés au niveau national et international, et elle explique le fonctionnement du système actuel d'admission. Ensuite, les acteurs principaux et leurs discours sont présentés, et la dynamique entre les deux discours est examinée. Cette analyse montre qu'il y a six points où l'ambiguïté créée par le mélange de discours de souveraineté et du discours de l'humanitaire devient particulièrement évidente. En conclusion, une proposition est faite pour le maintien de l'équilibre entre l'influence des deux groupes d'acteurs : le renforcement du rôle du système juridique. Il est proposé qu'en entérinant les droits garantis aux réfugiés par la Charte canadienne des droits et libertés et par les traités internationaux signés par le Canada, les juges peuvent jouer un rôle crucial pour l'assurance d'un système de protection des réfugiés qui est juste et en conformité avec les obligations internationales du pays. 